\title{
New Phase Difference Measurement Method for Non-Integer Number of Signal Periods Based on Multiple Cross-Correlations
}

\author{
Xianwu Sun ${ }^{1}$, Ting'ao Shen ${ }^{1,2, *}$ and Xiangjing $\mathrm{Mu}^{1}$ \\ ${ }^{1}$ Department of Management Science and Engineering, Logistical Engineering University, Chongqing 401311, China \\ ${ }^{2}$ Department of Information Engineering, Logistical Engineering University, Chongqing 401311, China
}

\begin{abstract}
In this paper, a new phase difference measurement method is proposed for non-integer number of signal periods based on multiple cross-correlations, which is derived from the error analysis of the correlation method. Firstly, threesegment signals with the same center frequency are chosen. Then, the Hilbert transformation is conducted on the selected three-segment signals to make it have 90 degree phase shift. And then, the correlation functions of the selected three signals and its phase shifted signals are computed respectively. Certain properties of correlation and sine functions are adopted to obtain the computational formula of phase difference. Theory analyses show that the measurement accuracy of the proposed method is not affected by whether the signal is in integer periods or not, even if the frequency is unknown. Simulations and comparisons are presented to validate the effectiveness of the proposed method.
\end{abstract}

Keywords: Phase difference measurement, Correlation method, Non-integer period signal, Hilbert transformation.

\section{INTRODUCTION}

Estimation of the phase difference between two noisy versions of the same signal received at two spatially separated sensors has attracted considerable attention in fault diagnosis, direction finding, electric power calibration, source localization and other fields [1-10].

In the past three decades, lots of phase difference measurement methods have been proposed to provide good performance [5-10]. Generalized cross correlator (GCC) [5] is a conventional approach to estimate the phase difference by locating the cross correlation peak of the filtered version of two received signals, and it has been proved that optimum performance can be attained when the signals and noises are Gaussian distributed. However, it shows bias if the correlation length is not in an integer number of period.

In [6], a quadrature delay estimator (QDE) has been proposed for phase difference estimation based on crosscorrelation method. The technique utilizes the in-phase and quadrature-phase components of one of the receiver output and provides a high-resolution phase-shift estimate. The QDE improves the accuracy of phase difference measurement in high SNR, but it also causes a bias in estimation when calculation with a non-integer number of signal periods. In [7] and [8], a modified method that utilizes all inphase and quadrature-phase components of the received signals is presented, called the unbiased QDE (UQDE). The advantage of UQDE is that it shows no bias, even if the correlation length is not in an integer number of period. However,

*Address correspondence to this author at the Logistical Engineering University, 401311, Chongqing, China; Tel: +15123209075;

E-mails: peoplesta@sina.com both QDE and UQDE show bias if the introduced phase delay is not exactly $\pi / 2$, and if the frequency of the signal is unknown, the methods will become invalid. In [9], the authors present a new method by applying the idea of data extension to make the data length equal or close to integer periods. This method improves the precision of correlation method evidently, but it fails to work if the frequency is unknown. In [10], a new method based on double correlation is proposed. This method improves the accuracy of phase difference measurement in low SNR, and it works even if the frequency is unknown. However, the disadvantage of this method is that it shows significant bias, if the correlation length is not in an integer number of period.

As is known for correlation methods, the whole data length is usually regarded as the correlation length to calculate the phase difference, which will lead correlation methods fail to work for dynamic phase difference measurement. If the data length is too long, the computational load of the correlation methods will increase a lot accordingly. What's more, most presented methods require a known frequency and the correlation length in an integer number of period. Based on the above analysis, it is important to propose a new method to overcome these problems and make it universality.

In this paper, a new method is proposed to remove the bias for unknown frequency under the non-integer number of signal periods. We firstly choose three-segment signals which have the same center frequency. Then, conduct the Hilbert transformation on the three-segment signals, and then adopt multiple cross-correlations operation among the signals. The phase difference is obtained by utilizing the properties of correlation and sine functions. We remark that the idea of this method is applicable to deal with other phase 
difference with unknown frequency, and the proposed method has higher measurement accuracy and stronger antidisturbance capability, even if the number of signal periods is not an integer.

The outline of this paper is organized as follows. Section 2 analyzes the error of correlation method. Then, a new phase difference measurement method for non-integer number of signal periods based on multiple cross-correlations is presented in Section 3. Numerical examples are presented in Section 4 to corroborate the theoretical development and to evaluate the performance of the proposed method. Finally, conclusions are drawn in Section 5.

\section{ERROR ANALYSIS OF CORRELATION METHOD}

Two real-valued sinusoids $x(t)$ and $y(t)$ without dc offset may be expressed as:

$$
\begin{aligned}
& x(t)=A \cos \left(\omega t+\theta_{1}\right)+N_{1}(t) \\
& y(t)=B \cos \left(\omega t+\theta_{2}\right)+N_{2}(t)
\end{aligned}
$$

where $\mathrm{A}$ and $\mathrm{B}$ are unknown tone amplitudes, $\omega$ is the unknown angular frequency, $\theta_{1}$ and $\theta_{2}$ are unknown initial phases, $N_{1}(t)$ and $N_{2}(t)$ are uncorrelated additive white Gaussian noise.

Calculate the correlation in the following range $(0, k T+\Delta T), T$ is the period of vibration signal, and $k$ is a positive integer. Then, the correlation calculations are given as

$$
\begin{aligned}
& R_{x y}(0)=\frac{1}{k T+\Delta T} \int_{0}^{k T+\Delta T} x(t) y(t) \mathrm{d} t \\
& =\frac{A B}{2} \cos \left(\theta_{2}-\theta_{1}\right)+\frac{A B}{2 \omega(k T+\Delta T)} \cos \left(\omega \Delta T+\theta_{1}+\theta_{2}\right) \sin (\omega \Delta T) \\
& R_{x x}(0)=\frac{1}{k T+\Delta T} \int_{0}^{k T+\Delta T} x(t) x(t) \mathrm{d} t \\
& =\frac{A^{2}}{2}+\frac{A^{2}}{2 \omega(k T+\Delta T)} \cos \left(\omega \Delta T+2 \theta_{1}\right) \sin (\omega \Delta T) \\
& R_{y y}(0)=\frac{1}{k T+\Delta T} \int_{0}^{k T+\Delta T} y(t) y(t) \mathrm{d} t \\
& =\frac{B^{2}}{2}+\frac{B^{2}}{2 \omega(k T+\Delta T)} \cos \left(\omega \Delta T+2 \theta_{2}\right) \sin (\omega \Delta T)
\end{aligned}
$$

Our goal is to evaluate the phase difference $\Delta \theta$ between these two sinusoids. $\Delta \theta$ can be given as:

$$
\Delta \theta=\theta_{2}-\theta_{1}=\arccos \left(\frac{2 R_{x y}(0)}{A B}\right)=\arccos \left(\frac{R_{x y}(0)}{\sqrt{R_{x x}(0)} \sqrt{R_{y y}(0)}}\right)
$$

Equation (5) is the expression of the cross-correlation method. It can be seen from (2) to (4) that if the correlation calculation range is not an integer number of period, the result shows bias. The phase difference calculation error depends on the $\Delta T$ equal to zero or not.

For discrete-time signals, the integral operation of correlation becomes multiplying and addition operation. The formulas from (2) to (4) can be expressed as:

$$
\begin{aligned}
& R_{x y}(0)=\frac{1}{N} \sum_{n=0}^{N-1} x(n) y(n)=\frac{A B}{2} \cos \left(\theta_{2}-\theta_{1}\right)+\frac{A B}{2 N} \sum_{n=0}^{N-1} \cos \left(2 \omega n+\theta_{1}+\theta_{2}\right) \\
& R_{x x}(0)=\frac{1}{N} \sum_{n=0}^{N-1} x^{2}(n)=\frac{A^{2}}{2}+\frac{A^{2}}{2 N} \sum_{n=0}^{N-1} \cos \left(2 \omega n+2 \theta_{1}\right) \\
& R_{y y}(0)=\frac{1}{N} \sum_{n=0}^{N-1} y^{2}(n)=\frac{B^{2}}{2}+\frac{B^{2}}{2 N} \sum_{n=0}^{N-1} \cos \left(2 \omega n+2 \theta_{2}\right)
\end{aligned}
$$

We can see from (6) to (8) that if the correlation calculation is not in one period or integer periods, the result of cross-correlation method shows bias, and the phase difference calculation error item has 2 times frequency as the vibration signal. When calculate the correlation in integer periods, this error part is zero. Thus, the main reason that caused the phase difference error of cross-correlation method is the number of signal periods is not in integer periods.

\section{PROPOSED METHOD}

To improve the precision of phase difference measurement with unknown frequency, and remove the bias under the non-integer number of signal periods, a new method is proposed in this paper. The main idea of the proposed method utilizes the Hilbert transformation and multiple crosscorrelations theory.

Firstly, three-segment signals $x(n), y(n)$ and $z(n)$ with the same frequency can be expressed as

$$
\begin{aligned}
& x(n)=A \cos \left(\omega n+\theta_{1}\right)+N_{1}(n) \\
& y(n)=B \cos \left(\omega n+\theta_{2}\right)+N_{2}(n) \\
& z(n)=C \cos \left(\omega n+\theta_{3}\right)+N_{3}(n)
\end{aligned}
$$

Note that the three-segment signals can be easily obtained from the original signals by using short rectangular window. Besides, we can also regard $x(n), y(n)$ and $z(n)$ as three individual signals detected by three transducers.

Then, the Hilbert transformation is conducted on the three-segment signals $x(n), y(n)$ and $z(n)$, which aims to make three-segment signals have 90 degree phase shift, as follows.

$$
\begin{aligned}
& x^{\prime}(n)=A \sin \left(\omega n+\theta_{1}\right)+N_{1}^{\prime}(n) \\
& y^{\prime}(n)=B \sin \left(\omega n+\theta_{2}\right)+N_{2}^{\prime}(n) \\
& z^{\prime}(n)=C \sin \left(\omega n+\theta_{3}\right)+N_{3}^{\prime}(n)
\end{aligned}
$$

And then, cross-correlations are computed between $x(n)$, $y(n), x^{\prime}(n), y^{\prime}(n)$ and $z(n), z^{\prime}(n)$ respectively, as follows. 
In ideal condition, the noise signal is not correlated to vibration signal, and noise signal is not correlated to each other. So, (11) can be defined as follows:

$$
\begin{aligned}
& R_{x z}(0)=\frac{1}{N} \sum_{n=0}^{N-1} x(n) z(n) ; R_{x^{\prime} z^{\prime}}(0)=\frac{1}{N} \sum_{n=0}^{N-1} x^{\prime}(n) z^{\prime}(n) \\
& R_{x z^{\prime}}(0)=\frac{1}{N} \sum_{n=0}^{N-1} x(n) z^{\prime}(n) ; R_{x^{\prime} z}(0)=\frac{1}{N} \sum_{n=0}^{N-1} x^{\prime}(n) z(n) \\
& R_{y z}(0)=\frac{1}{N} \sum_{n=0}^{N-1} y(n) z(n) ; R_{y^{\prime} z^{\prime}}(0)=\frac{1}{N} \sum_{n=0}^{N-1} y^{\prime}(n) z^{\prime}(n) \\
& R_{y z^{\prime}}(0)=\frac{1}{N} \sum_{n=0}^{N-1} y(n) z^{\prime}(n) ; R_{y^{\prime} z}(0)=\frac{1}{N} \sum_{n=0}^{N-1} y^{\prime}(n) z(n) \\
& { }_{n=0}
\end{aligned}
$$$$
R_{x z}(0)=\frac{A C}{2} \cos \left(\theta_{3}-\theta_{1}\right)+\frac{A C}{2 N} \sum_{n=0}^{N-1} \cos \left(2 \omega n+\theta_{1}+\theta_{3}\right)
$$$$
R_{x^{\prime} z^{\prime}}(0)=\frac{A C}{2} \cos \left(\theta_{3}-\theta_{1}\right)-\frac{A C}{2 N} \sum_{n=0}^{N-1} \cos \left(2 \omega n+\theta_{1}+\theta_{3}\right)
$$$$
R_{x z^{\prime}}(0)=\frac{A C}{2} \sin \left(\theta_{3}-\theta_{1}\right)+\frac{A C}{2 N} \sum_{n=0}^{N-1} \sin \left(2 \omega n+\theta_{1}+\theta_{3}\right)
$$$$
R_{x^{\prime} z}(0)=-\frac{A C}{2} \sin \left(\theta_{3}-\theta_{1}\right)+\frac{A C}{2 N} \sum_{n=0}^{N-1} \sin \left(2 \omega n+\theta_{1}+\theta_{3}\right)
$$$$
R_{y z}(0)=\frac{B C}{2} \cos \left(\theta_{3}-\theta_{2}\right)+\frac{B C}{2 N} \sum_{n=0}^{N-1} \cos \left(2 \omega n+\theta_{2}+\theta_{3}\right)
$$$$
R_{y^{\prime} z^{\prime}}(0)=\frac{B C}{2} \cos \left(\theta_{3}-\theta_{2}\right)+\frac{B C}{2 N} \sum_{n=0}^{N-1} \cos \left(2 \omega n+\theta_{2}+\theta_{3}\right)
$$$$
R_{y z^{\prime}}(0)=\frac{B C}{2} \sin \left(\theta_{3}-\theta_{2}\right)+\frac{B C}{2 N} \sum_{n=0}^{N-1} \sin \left(2 \omega n+\theta_{2}+\theta_{3}\right)
$$$$
R_{y^{\prime} z}(0)=-\frac{B C}{2} \sin \left(\theta_{3}-\theta_{2}\right)+\frac{B C}{2 N} \sum_{n=0}^{N-1} \sin \left(2 \omega n+\theta_{2}+\theta_{3}\right)
$$

According to (12), we obtain

$$
\begin{aligned}
& R_{x z}(0)+R_{x^{\prime} z^{\prime}}(0)=A C \cos \left(\theta_{3}-\theta_{1}\right) \\
& R_{x z^{\prime}}(0)-R_{x^{\prime} z}(0)=A C \sin \left(\theta_{3}-\theta_{1}\right) \\
& R_{y z}(0)+R_{y^{\prime} z^{\prime}}(0)=B C \cos \left(\theta_{3}-\theta_{2}\right) \\
& R_{y z^{\prime}}(0)-R_{y^{\prime} z}(0)=B C \sin \left(\theta_{3}-\theta_{2}\right)
\end{aligned}
$$

Utilize the properties of sine functions as follows.

$$
\begin{aligned}
& \sin \left(\theta_{2}-\theta_{1}\right)=\sin \left[\left(\theta_{3}-\theta_{1}\right)-\left(\theta_{3}-\theta_{2}\right)\right] \\
& =\sin \left(\theta_{3}-\theta_{1}\right) \cos \left(\theta_{3}-\theta_{2}\right)-\cos \left(\theta_{3}-\theta_{1}\right) \sin \left(\theta_{3}-\theta_{2}\right) \\
& =\frac{\left(R_{x z^{\prime}}-R_{x^{\prime} z}\right)}{A C} \cdot \frac{\left(R_{y z}+R_{y z^{\prime}}\right)}{B C}-\frac{\left(R_{x z}+R_{x z^{\prime}}\right)}{A C} \cdot \frac{\left(R_{y z^{\prime}}-R_{y y^{\prime} z}\right)}{B C} \\
& \cos \left(\theta_{2}-\theta_{1}\right)=\cos \left[\left(\theta_{3}-\theta_{1}\right)-\left(\theta_{3}-\theta_{2}\right)\right] \\
& =\cos \left(\theta_{3}-\theta_{1}\right) \cos \left(\theta_{3}-\theta_{2}\right)+\sin \left(\theta_{3}-\theta_{1}\right) \sin \left(\theta_{3}-\theta_{2}\right) \\
& =\frac{\left(R_{x z}+R_{x^{\prime} z^{\prime}}\right)}{A C} \cdot \frac{\left(R_{y z}+R_{y z^{\prime}}\right)}{B C}+\frac{\left(R_{x z^{\prime}}-R_{x^{\prime} z^{\prime}}\right)}{A C} \cdot \frac{\left(R_{y z^{\prime}}-R_{y z}\right)}{B C}
\end{aligned}
$$

$$
\begin{aligned}
\tan \left(\theta_{2}\right. & \left.-\theta_{1}\right)=\frac{\sin \left(\theta_{2}-\theta_{1}\right)}{\cos \left(\theta_{2}-\theta_{1}\right)} \\
= & \frac{\left(R_{x z^{\prime}}-R_{x^{\prime} z}\right)\left(R_{y z}+R_{y^{\prime} z^{\prime}}\right)-\left(R_{x z}+R_{x^{\prime} z^{\prime}}\right)\left(R_{y z^{\prime}}-R_{y^{\prime} z}\right)}{\left(R_{x z}+R_{x^{\prime} z^{\prime}}\right)\left(R_{y z}+R_{y^{\prime} z^{\prime}}\right)+\left(R_{x z^{\prime}}-R_{x^{\prime} z}\right)\left(R_{y z^{\prime}}-R_{y^{\prime} z}\right)}
\end{aligned}
$$

Therefore, the phase difference $\Delta \theta$ can be obtained as

$$
\Delta \theta=\theta_{2}-\theta_{1}=\arctan \frac{\left(R_{x z^{\prime}}-R_{x^{\prime} z}\right)\left(R_{y z}+R_{y z^{\prime}}\right)-\left(R_{x z}+R_{x z^{\prime}}\right)\left(R_{y z^{\prime}}-R_{y z}\right)}{\left(R_{x z}+R_{x^{\prime} z}\right)\left(R_{y z}+R_{y z^{\prime}}\right)+\left(R_{x z^{\prime}}-R_{x^{\prime} z}\right)\left(R_{y z^{\prime}}-R_{y^{\prime} z}\right)}
$$

From (17), we conclude that even if the frequency is unknown, the phase difference of the proposed method can be obtained in high accuracy, without knowing the amplitudes of the segment signals. What's more, the proposed method shows no bias, even if the number of signal periods is not an integer.

The double correlation method presented in [10] can be obtained as follows

$$
\Delta \theta=\tan ^{-1}\left(\frac{R_{x y^{\prime}}(0)}{R_{x y}(0)}\right)
$$

From (18), we can see that it works even if the frequency is unknown. But it shows bias, if the number of signal periods is not an integer.

\section{SIMULATION RESULTS}

In this section, simulation experiments are presented to ascertain the performance of the proposed method. The proposed method is compared with cross-correlation method and double correlation method. The performances of the three methods are evaluated in white Gaussian noise with zero mean. We use Root Mean Square Error (RMSE) to evaluate the performance of the three methods, and each RMSE tests from 100 Monte Carlo trials. In simulations, the signal frequency and the sampling frequency are set to 100 $\mathrm{Hz}$ and $2000 \mathrm{~Hz}$, respectively.

Firstly, we compare the RMSE of the three methods with respect to the SNR, and the SNR varies from $0 \mathrm{~dB}$ to $40 \mathrm{~dB}$. Fig. (1) shows the RMSE of the three methods under the integer number of signal periods, such as $\mathrm{N}=20$. While Fig. 2 shows the RMSE of the three methods under the non-integer number of signal periods, such as $\mathrm{N}=15$.

In Fig. 1, when the number of signal periods is an integer, the accuracy of the proposed method is superior to cross-correlation method and double correlation method in different SNR, especially in low SNR, because the proposed method adopts the multiple cross-correlations, which markedly decreases the effect of the noises. In Fig. 2, when the number of signal periods is not an integer, the accuracy of cross-correlation method and double correlation method show serious bias, while the accuracy of the proposed method changes hardly.

From Fig. (1 and 2), we can see that the RMSE curves of the three methods decrease as SNR increases. The RMSE curve of the proposed method locates below that of cross- 


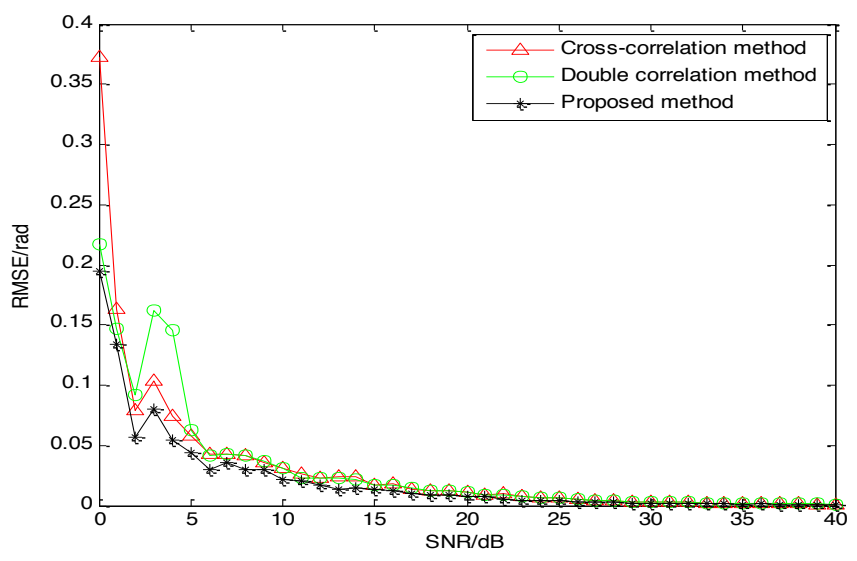

Fig. (1). RMSE comparison with different $S N R$ at $N=20$.

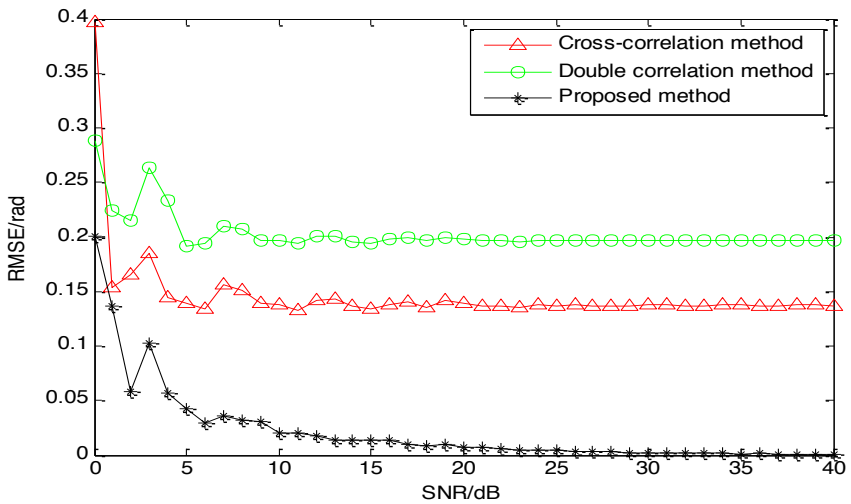

Fig. (2). RMSE comparison with different $S N R$ at $N=15$.

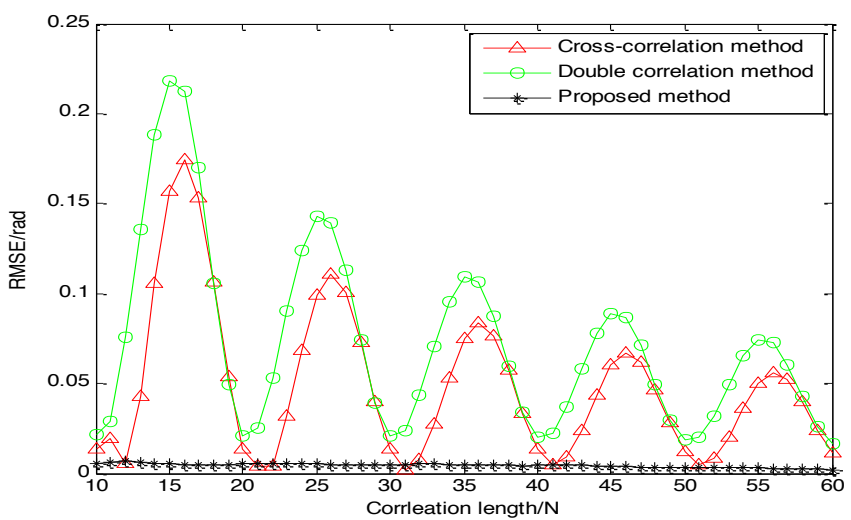

Fig. (3). RMSE comparison with different correlation length.

correlation method and double correlation method all long, which indicates that the proposed method has higher measurement accuracy and stronger anti-disturbance capability than the other two methods in different SNR. Besides, we can see that cross-correlation method and double correlation method work well only in an integer number of signal periods, while the proposed method is not affected by whether integer periods or not, which indicates that the proposed method has good universality.

In Fig. (3), we investigate the performance of the three methods against different correlation length. The SNR is fixed at $20 \mathrm{~dB}$. The correlation length ranges 10 to 60 . As can be seen, the accuracy of cross-correlation method and double correlation method are affected by correlation length, while the proposed method is not. In other words, the proposed method is not affected by whether integer periods or not, while the other two methods are not.

We can also notice a feature of the proposed method from (17), that it has no bias for number of samples, including being applied to only two samples for each sinusoid. Thus, the proposed method can be applied to calculate dynamic phase difference. This is a peculiarly character which other correlation methods don't have. In Fig. 4, we just test 


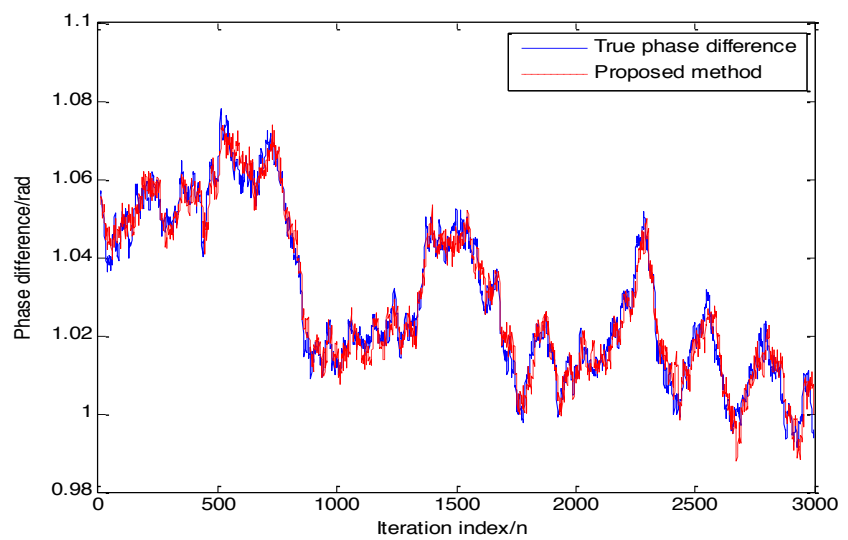

Fig. (4). Dynamic phase difference estimated by proposed method.

the dynamic performance of the proposed method, for the other correlation methods cannot track the dynamic phase difference. The SNR and correlation length are set to $20 \mathrm{~dB}$ and 2, respectively. We can see that the proposed method works well, and the result validates the dynamic performance of the proposed method.

\section{CONCLUSIONS}

A new phase difference measurement method is presented for non-integer number of signal periods based on multiple cross-correlations in this paper, and the proposed method has three attractive features. First, if the frequency is unknown, the proposed method is also effective. Second, the proposed method shows no bias, even if the number of signal periods is not an integer. Third, the proposed method can be applied to calculate dynamic phase difference, for it has no bias for number of samples, including being applied to only two samples for each sinusoid. The proposed method has higher measurement accuracy and stronger anti-disturbance capability. The simulation results demonstrate the performance of the proposed method.

\section{CONFLICT OF INTEREST}

The authors confirm that this article content has no conflicts of interest.

\section{ACKNOWLEDGMENT}

The authors would like to thank the Associate Editor and the reviewers for their helpful comments, which improved the quality of this paper.

\section{REFERENCES}

[1] H.C. So, "Time-delay estimation for sinusoidal signals" IEE Proceedings on Radar, Sonar and Navigation, vol. 148, no. 6, pp. 318-324, 2001.

[2] M. Chakraborty, H.C. So and J. Zheng, "New adaptive algorithm for delay estimation of sinusoidal signals", IEEE Signal Processing Letters, 14(12): 984-987, 2007.

[3] T.A. Shen, Y.Q. Tu and H.T. Zhang, "A novel time varying signal processing method for Coriolis mass flowmeter", Review of Scientific Instruments, vol. 85, no. 6, pp. 065116, 2014.

[4] X. Nie and L.P. Li, "A computationally efficient subspace algorithm for 2-D DOA estimation with L-shaped array". IEEE Signal Processing Letters, 21(8): 971-974, 2014.

[5] C.H. Knapp and G.C. Carter, "The generalized correlation method foe estimation of time delay", IEEE Transactions on Acoustics, Speech and Signal Processing, vol. 24, no. 4, pp. 320-327, 1976.

[6] D.L. Maskell and G.S. Woods, "The discrete-time quadrature subsample estimation of delay" IEEE Transaction on Instrumentation and Measurement, vol. 51, no. 1, pp. 133-137, 2002.

[7] H.C. So, "A comparative study of two discrete-time phase delay estimators," IEEE Transaction on Instrumentation and Measurement, vol. 54, no. 6, pp. 2501-2504, 2005.

[8] M.V. Nada and V.S. Lazar, "A simple algorithm for the estimation of phase difference between two sinusoidal voltages" IEEE Transaction on Instrumentation and Measurement, vol. 59, no. 12, pp. 31523158, 2010.

[9] T.A. Shen, Y.Q. Tu, M. Li, and H.T. Zhang, "Research and validation on improved correlation method for phase difference measurement based on data extension" Chinese Journal of Scientific Instrument, vol. 35, no. 6, pp. 1331-1337, 2014.

[10] J.M. Li, P. Zhao, and W. Hou, "Research on error of phase difference algorithm based on correlation theory" Journal of North University of China (Natural Science Edition), vol. 30, no. 6, pp. 616- 619, 2009.

Received: May 26, 2015

Revised: July 14, 2015

Accepted: August 10, 2015

(C) Sun et al.; Licensee Bentham Open.

This is an open access articles licensed under the terms of the Creative Commons Attribution-Non-Commercial 4.0 International Public License (CC BY-NC 4.0) (https://creativecommons.org/licenses/by-nc/4.0/legalcode), which permits unrestricted, non-commercial use, distribution and reproduction in any medium, provided that the work is properly cited. 